feministische
Hect2,2019
studien

Inhaltsverzeichnis

\section{Cyborgs Revisited: Zur Verbindung von Geschlecht, Technologien und Maschinen}

\author{
Anna Lena Berscheid, Ilona Horwath und \\ Birgitt Riegraf \\ Einleitung 241
}

Katharina Hoppe

Donna Haraways Gefährt`innen:

Zur Ethik und Politik der

Verwobenheit von Technologien,

Geschlecht und Ökologie 250

Katharina Liebsch

Zwischen Science Fantasy und ontologischer Politik. Künstlerischer

Technomaterialismus jenseits der

Geschlechtergrenzen 269

Julia Feiler

Risiko und Verantwortung im Umgang mit alternden Eizellen.

'Social Freezing`als Sozialtechnik

des Zukunftsmanagements $\mathbf{2 8 9}$

\section{Bianca Prietl}

Algorithmische Entscheidungssysteme revisited: Wie Maschinen gesellschaftliche Herrschaftsverhältnisse reproduzieren können 303

Dag Balmar and Ulf Mellström

Cyborgs and entanglements of technology, masculinity, and (automated) vehicles $\mathbf{3 2 0}$

\section{Im Gespräch}

Donna Haraway und Thyrza Nichols Goodeve Speaking Resurgence to Despair/

I'd Rather Stay With the Trouble 335

\section{Bilder und Zeichen}

Tone Bjordam

Natur, Gesellschaft, Wissenschaft 348

\section{Diskussion}

Tanja Kubes

Sexroboter - Queerfeministisches

Potential oder materialisierte

Objektifizierung? 351

\section{Bericht aus laufender Forschung}

Edelgard Kutzner und Melanie Roski Arbeit, Technik und Geschlecht neue Grenzziehungen durch

Digitalisierung? 363

\section{Tagungsberichte}

Nadine Dannenberg und Myriam Raboldt Difference, Diversity, Diffraction:

Confronting Hegemonies and

Dispossessions.

10th European Feminist Research

Conference in Göttingen,

12.-15. September 2018373

Francis Seeck und Cash Hauke

Gender-Care - Migration.

Ambivalente Interdependenzen.

30. Januar bis 1. Februar 2019,

Humboldt-Universität zu Berlin 376

Susanne Lettow

Contested Kinship.

Towards a Redefinition of Human

Relations.

Internationale Konferenz, Georg-

August-Universität Göttingen, 14.-16. März 2019379 
Rezensionen

Sybilla Flügge

Ute Gerhard: Für eine andere

Gerechtigkeit. Dimensionen

feministischer Rechtskritik 383

Julia Gruhlich

Eva Senghaas-Knobloch: Arbeit ist

keine Ware - 100 Jahre Internationale

Arbeitsorganisation 385

Sandra Beaufä̈s

Sabine Hark/Johanna Hofbauer (Hrsg.):

Vermessene Räume, gespannte

Beziehungen. Unternehmerische

Universitäten und

Geschlechterdynamiken $\mathbf{3 8 8}$

Anna-Lena Berscheid

Beate Kortendiek/Birgit Riegraf/

Katja Sabisch (Hrsg.):

Handbuch interdisziplinäre

Geschlechterforschung 391
Annika Spilker

Ortrud Wörner-Heil: Käthe Delius

(1893-1977). Hauswirtschaft als

Wissenschaft 393

Zu den Autor_innen 396

Abstracts 399

Jahresinhaltsverzeichnis 403

Bestellformular 409

Förderverein 410

Die nächsten Hefte 411

Die Zeitschrift und alle in ihr enthaltenen einzelnen Beiträge werden regelmäßig von Social Science Citation Index, Social SciSearch und Journal Citation Reports / Social Sciences Edition, dem Sozialwissenschaftlichen Literaturinformationssystem SOLIS und dem Zeitschriftendienst der Deutschen Nationalbibliothek erfasst.

Die Zeitschrift und alle in ihr enthaltenen Beiträge und Abbildungen sind urheberrechtlich geschützt. Jede Verwendung außerhalb der engen Grenzen des Urheberrechtsgesetzes ist ohne Zustimmung des Verlages unzulässig und strafbar. Das gilt insbesondere für Vervielfältigungen, Übersetzungen, Mikroverfilmungen und die Einspeicherung und Verarbeitung in elektronischen Systemen. 